\title{
Improvements to In Vitro Culture Media for Use in Bovine IVF
}

\author{
Do $\mathrm{VH}^{1,2}$, Walton $\mathrm{S}^{3}$ and Taylor-Robinson $\mathrm{AW}^{* 1}$
}

${ }^{1}$ School of Medical \& Applied Sciences, Central Queensland University, Rockhampton, QLD, Australia

${ }^{2}$ National Key Laboratory of Animal Cell Technology, National Institute of Animal Sciences, Hanoi, Vietnam

${ }^{3}$ Australian Reproductive Technologies, Mt Chalmers, QLD, Australia

${ }^{*}$ Corresponding author: Taylor-Robinson AW, School of Medical \& Applied Sciences, Central Queensland University, Bruce Highway, Rockhampton, QLD 4702, Australia, Tel: 61749232008, E-mail: a.taylorrobinson@cqu.edu.au

Citation: Do VH, Walton S, Taylor-Robinson AW (2016) Improvements to In Vitro Culture Media for Use in Bovine IVF. J Vet Sci Anim Husb 4(2): 205. doi: 10.15744/2348-9790.4.205

Received Date: April 21, 2016 Accepted Date: May 25, 2016 Published Date: May 27, 2016

\begin{abstract}
In assisted reproduction of cattle the design and preparation of in vitro culture media has been instrumental in supporting the development of bovine oocytes and embryos. In vitro production (IVP) involves three main sequential steps: oocyte aspiration and in vitro maturation (IVM); in vitro fertilization (IVF); and in vitro culture (IVC) of early pre-implantation embryos. Research on IVC media has aimed to emulate the crucial elements that are present in the oviduct and uterus of female cattle by supplementing with protein and growth factors. As a result, IVP has proven a reliable methodology with an acceptable, if far from remarkable, blastocyst transfer rate of typically $40 \%$. Moreover, the addition to media of defined extracts has gradually replaced that of fetal calf serum, leading to improved embryo viability and cryotolerance. Nevertheless, major advances in the composition of novel media that may facilitate a further increase of blastocyst rate have yet to be realized. Currently, there is considerable variation in the constituents of IVC media used in bovine assisted reproduction. Hence, significant innovations are required in order to not only achieve a greater success rate of IVP and to enhance the cryotolerance of bovine IVF-derived embryos but also to provide a defined IVC medium that may be recognized as a point of reference across the cattle breeding industry. This review examines the progress made to date in IVP to enhance embryo quality and points to future areas for investigation of a 'gold standard' IVC medium. This is a fully defined medium that supports consistently the attainment of a bovine IVP blastocyst rate similar to that achieved using a conventional medium supplemented with serum.
\end{abstract}

Keywords: in vitro; IVF; Culture; Oocyte; Embryo; Blastocyst; Oviduct; Bovine

\section{Introduction}

The emerging trend in bovine assisted reproduction in recent years is that in vitro production (IVP) is increasingly overtaking multi-ovulation embryo transfer as the technology of choice in several countries, including Brazil [1-3]. Moreover, Parrish [4] stated that bovine IVP has become a competitive alternative to multiple ovulation embryo transfer (MOET) and plays an important role in commercial settings. The reasons include the coupling of IVP with ovum pick-up (OPU) from live cows that enables aspiration of a large number of ovarian follicles from Bos indicus cattle oocytes without the stimulation of gonadotropin hormones [5]. In addition, highly transferable IVP embryos correlate with cattle possessing considerable numbers of ovarian follicles [6]. In addition, Pontes et al. [5] reported that the number of IVP embryos was greater than those attained by in vivo production over the same period of time. Moreover, Taylor-Robinson et al. [3] contended that IVP may increase genetic gain in a breeding herd, as OPU can be performed with young cattle [7]. Furthermore, there is no doubt that bovine in vitro fertilization (IVF) technology is an important research tool which may be used in order to understand early embryo development [8].

In terms of embryo production costs, Galli et al. [2] noted that for dairy cattle in Europe the cost of an IVP embryo is 1.5-2 times that of an in vivo embryo, leading to a modest demand; nevertheless, the costs of IVP in beef is still cheaper than MOET. Preselecting the gender of offspring through IVF with sex-sorted sperm can reduce the cost of embryo production because single sex-sorted semen straws can fertilize several cows in just one dish of the IVF system $[9,10]$. As a result, IVF technology that uses sex-sorted sperm is proven to be feasible under commercial conditions [10].

Despite such advantages over MOET, IVF remains a relatively inefficient process with blastocyst rates of around 40\% [11,12]. This may be due to the fact that the support provided by the in vitro culture (IVC) system to oocytes subjected to IVP remains sub-optimal. IVP systems possibly lack physiological pathways and essential components that allow the development of bovine embryos [8]. 
Moreover, worldwide 90\% of IVP embryos are transferred fresh, reflecting their lower cryotolerance [13]. Hence, modification of embryo culture media is necessary to improve the survival rates of IVP embryos [14]. Considerable attempts have been made to improve the yield of transferred blastocysts and their cryotolerance by supplementing IVC media with various growth factors and compounds thought to be stimulating [15-17]. Furthermore, research has focused attention on designing and establishing a novel defined culture medium that can replace serum under IVC conditions.

The purpose of this short review is to describe recent efforts to develop IVF systems that aim to enhance the blastocyst production and cryotolerance of IVP embryos. Significant improvements to and/or innovations in IVC conditions could make the use of IVF both more efficient and accessible and hence widespread in the cattle industry.

\section{Variations of In Vitro Culture Media}

An experimental approach has been successful in designing and generating various culture media based on an understanding of embryonic development in the female reproductive tract. Ovum-pick up technique allows the collection of bovine immature oocytes without the stimulation of gonadotropin hormones. These immature oocytes are then subjected to in vitro culture when they are influenced by various proteins and growth factors [18]. The most popular in vitro maturation (IVM) medium to use to support bovine oocyte in vitro maturation is tissue culture medium 199 (TCM199) supplemented with gonadotropin hormones and fetal calf serum (FCS). A study of the supplementation of TCM199 medium with tretinoin and lipid-core nanocapsules reported a blastocyst rate of 46.8\% [19]. Based on TCM199 medium, Machado et al. [20] added fibroblast growth factor 17 alone or combination with bone morphogenetic protein 15 to IVM medium, which had the effect of increasing the expansion of cumulus cells, leading to an increase in blastocyst rates to around 50\%. Bone morphogenetic protein 15 and fibroblast growth factor both regulate the function of cumulus cells and enhance embryo quality [20].

Typically, after the maturation of bovine oocytes in IVM for 22-24 hours, oocytes are subjected to fertilization. Two commonly used IVF media are Brackett-Oliphant medium and 4-(2-hydroxyethyl)-1-piperazineethanesulfonic acid) (HEPES)-buffered Tyrode's albumin lactate pyruvate (TALP) containing heparin. Tajik and Niwa [21] pointed out the synergistic effect of heparin and caffeine in IVF medium for spermatozoa capacitation. Monaco et al. [22], supplementing osteopontin in TALP medium, attained 56.4\% blastocyst rates.

The success of embryo production is dependent not only on IVM and IVF media but also IVC medium. The ubiquitous IVC media currently used are CRlaa and modified synthetic oviduct fluid (SOF) supplemented with FCS and amino acids. The oviduct and uterus contain assorted specific regulatory elements, such as hormones, growth factors and cytokines, which when added to culture media are capable of enhancing the development of IVP pre-implantation embryos [14]. Neira et al. [16], testing the association of a wide range of governing factors (insulin-like growth factors I and II, basic fibroblast growth factor, transforming growth factors, granulocyte macrophage colony-stimulating factor, and leukemia inhibitory factor), indicated that supplementation of these components to IVC medium increased the yield of transferred blastocysts. In another study, Lim et al. [15] supplemented IVC medium with epidermal growth factor and reported a high blastocyst rate of 51.7\% (108/261).

It is important to note that the supplementation of unusual components such as leptin [23], copper sulphate [24] and exogenous glutathione [25] to IVC has achieved better results. However, on some occasions the addition of factors like melatonin to IVM medium did not improve embryo production [26]. Another example is the addition to IVC medium of dexamethasone, which has the effect of raising the embryonic cell number but does not lessen the apoptosis rate of IVP embryos [27].

Furthermore, serum is a common supplement to IVC media, for which the concentration routinely added is $10 \% \mathrm{v} / \mathrm{v}[28,29]$. FCS consists of 60-80 g protein (of which albumin accounts for 60\%), growth factors, energy metabolites including glucose, amino acids and vitamins (Catt S, personal communication). Therefore, FCS is an important component that supports the maturation of oocytes and subsequent development of embryos [30]. In corroboration, FCS improves cell proliferation and assists blastocyst hatching [16]. Nevertheless, the presence of FCS in media increases the lipid content of IVP embryos [31,32], causing a detrimental effect on their cryosurvival. Thus, a reduction of serum level to $2 \% \mathrm{v} / \mathrm{v}$ in IVC medium is necessary to maintain blastocyst yield and embryo quality [33]. Moreover, supplementing IVC media with FCS can lead to inconsistent results between different IVP sessions [18], due to the intrinsically variable composition of FCS [16].

\section{Development of Defined In Vitro Culture Media}

The aim of preparing defined media is to minimize the variation in unknown components that occurs naturally in serum. This has the advantage of providing a simplified technique that enhances cryoresistance of embryos. This is due to avoiding the supplementation of biological components such as FCS and bovine serum albumin (BSA) that may increase the potential risks of contamination [16].

Bone morphogenetic protein 15 and fibroblast growth factor regulate the function of cumulus cells and increase embryo quality [20]. In spite of this, FCS is still often used in IVM medium, contrary to the rationale that defined media are beneficial for reasons of biosafety and identification of standardized constituents [34,35]. Evidence shows that use of defined media arrests the nuclear maturity of oocytes cultured for 24 hours [36]. 
Currently, a dependency on the addition of sera continues since an innovative medium that provides a complete substitute for IVM has yet to be introduced for use in bovine IVP. Previously, Holm et al. [30] attained a high percentage of blastocysts from defined IVC medium; however, they concluded that FCS is essential for IVM. Lee et al. [37] developed a defined IVC medium by supplementing with non-essential and essential amino acids, but the blastocyst rates in this study did not reach above $40 \%$. In addition, Neira et al. [16] designed a fully defined culture medium with the addition of a range of recombinant growth factors and cytokines; however, the blastocyst rates (ranging from 20 to 48\%) that resulted from using the defined medium were no greater than those from non-defined culture medium supplemented with FCS.

It may therefore be concluded that semi-defined in vitro media have been prepared successfully but that a fully defined in vitro medium remains to be formulated.

\section{Cryopreservation of IVP Embryos}

Commensurate with the advancement in cryopreservation technology, a modification to IVC media is critical in order to improve the survival rates of IVP embryos post cryopreservation [38]. Supplementation of silk protein sericin in the freezing medium but without serum results in similar survival and hatching rates of IVP embryos to those of freezing medium containing serum [35]. Addition of Albumax 1 (lipid-rich BSA) to freezing medium also achieved a better survival of IVP blastocysts (87.3\%) and calving rate $(22.6 \%)$ compared to rates of survival (70.9\%) and calving (10\%) when using freezing medium containing fetal bovine serum [39]. Nevertheless, the freezing of IVP embryos represents a considerable challenge [5]. Vitrification offers a promising alternative cryopreservation technique for such sensitive cells $[38,40]$. For example, Sanches et al. [40] reported a high mean pregnancy rate of $48.8 \%$ after the transfer of vitrified embryos.

Indeed, the quality of IVP embryos is lower than that of in vivo counterparts [41], in vivo embryos showing less lipid accumulation, low apoptotic index and better cryotolerance than do IVP embryos [42]. Moreover, it is thought that the main difficulty encountered in freezing IVP embryos is due to excessive accumulation of intracytoplasmic lipid droplets [40-43]. The restoration of IVP embryos after cryopreservation is hampered as lipid droplets affect cellular repair [44]. Consequently, efforts to enhance the quality of IVP embryos prior to their cryopreservation have been made by modification of IVC media. It is evident that those IVP blastocysts considered to be of excellent quality, 'grade A', had a greater cryosurvival than those classed as 'grade B', of good quality (57.2\% versus $41.7 \%$ ) [45]. Consistent with this, supplementation of the naturally occurring antioxidant L-ergothioneine to IVC medium improves embryo quality and hence promotes an increase in the hatching rates of IVP embryos following cryopreservation [17].

The presence of serum in media is the main cause of increased lipid content of IVP embryos [31,43]. Thus, the main purpose of the improvement in the quality of IVP embryos before cryopreservation is to reduce or eliminate totally the need to use FCS in culture media.

Sudano et al. [32] found that for IVC a reduction of FCS to $2.5 \% \mathrm{v} / \mathrm{v}$ and addition of phenazine ethosulfate, thereby inhibiting fatty acid synthesis, improves the cryotolerance of IVP embryos. In addition, IVP bovine embryos cultured in this medium without FCS showed greater survival rates after cryopreservation than those cultured in medium with FCS [44]. For example, Gomez et al. [45] reported that IVP blastocysts cultured in SOF medium plus BSA without FCS showed a higher survival rate after vitrification than those cultured in SOF with FCS (56.6\% and 35.2\%, respectively). Furthermore, Block et al. [46] indicated that supplementation of hyaluronan to SOF medium improved the bovine IVP survival rate following vitrification.

\section{Bovine IVP Using Sex-Sorted Sperm}

IVF technology provides the fertilization efficiency of sex-sorted sperm [9]. Artificial insemination with sex-sorted sperm shows a lower rate of pregnancy loss when compared to IVP with sex-sorted sperm, suggesting that IVC media require improvement in order to mimic closely in vivo conditions [47]. Ghys et al. [48] showed that female IVP embryos were more apoptotic than their male counterparts. They also found that apoptosis was compounded when embryos were cultured in IVC with $5 \% \mathrm{v} / \mathrm{v}$ FCS compared to IVC containing BSA, insulin, transferrin and selenium. In fact, IVP systems for sex-sorted sperm have not been specialized for resultant embryos. However, Liu et al. [49] indicated that the quality of sex-sorted semen is lower than that of conventional semen. In addition, the reduced efficacy of sex-predetermined IVP is due to the low fertility of sperm processed by flow cytometry [10], as well as to individual variations in bull fertility [49-51]. Recently, Li et al. [52] have reported that isolation of motile sex-sorted sperm by use of a microfluidic sperm sorter resulted in a blastocyst rate of around $40 \%$, which is similar to blastocyst yields achieved with non sex-sorted sperm.

\section{Conclusion}

In order to improve the generation of bovine IVP embryos many attempts have been made to develop a suitably supportive IVC medium by the addition of various factors. However, production of bovine embryos by IVF remains sub-optimal. The elimination of batch-to-batch variations in IVP by replacing sera with a novel defined IVC medium has not been fulfilled. To an extent reduction of the use of sera in preparing defined media enhances the cryotolerance of IVP embryos during the cooling and warming processes. Nonetheless, at present the IVP blastocyst yield that is achieved using defined IVC media is unsatisfactory; consequently, the 
replacement of serum in media by synthetic extracts remains a practical difficulty. Cryopreservation of IVP embryos still poses a technical challenge, as their survival rates after cryopreservation are lower than those of in vivo counterparts. IVP with semen has to overcome the motility of sex-sorted sperm. These obstacles may be resolved by further investigation of IVC media and related in vitro preparatory techniques. This has the potential to achieve a significant increase in the efficacy of IVP, which, in turn, would lead to its more extensive application in assisted reproduction by the cattle industry.

\section{Acknowledgement}

Dr Sally Catt of the Education Program in Reproduction \& Development, Monash Medical Centre, Clayton, Australia, is thanked for providing constructive comments on this manuscript.

\section{References}

1. Camargo LS, Freitas C, de Sa WF, de Moraes Ferreira A, Serapiao RV, et al. (2010) Gestation length, birth weight and offspring gender ratio of in vitro-produced Gyr (Bos indicus) cattle embryos. Anim Reprod Sci 120: 10-5.

2. Galli C, Duchia R, Colleoni S, Lagutina I, Lazzari G (2014) Ovum pick up, intracytoplasmic sperm injection and somatic cell nuclear transfer in cattle, buffalo and horses: from the research laboratory to clinical practice. Theriogenology 81: 138-51.

3. Taylor-Robinson AW, Walton S, Swain DL, Walsh KB, Vajta G (2014) The potential for modification in cloning and vitrification technology to enhance genetic progress in beef cattle in Northern Australia. Animal Reproduction Science 148: 91-6.

4. Parrish JJ (2014) Bovine in vitro fertilization: in vitro oocyte maturation and sperm capacitation with heparin. Theriogenology 81: 67-73.

5. Pontes JH, Nonato-Junior I, Sanches BV, Ereno-Junior JC, Uvo S, et al. (2009) Comparison of embryo yield and pregnancy rate between in vivo and in vitro methods in the same Nelore (Bos indicus) donor cows. Theriogenology 71: 690-7.

6. Santos GM, Silva-Santos KC, Barreiros TR, Morotti F, Sanches BV, et al. (2016) High numbers of antral follicles are positively associated with in vitro embryo production but not the conception rate for FTAI in Nelore cattle. Anim Reprod Sci 165: 17-21.

7. Kruip TA, Boni R, Wurth YA, Roelofsen MW, Pieterse MC (1994) Potential use of ovum pick-up for embryo production and breeding in cattle. Theriogenology 42: 675-84.

8. Besenfelder U, Havlicek V, Kuzmany A, Brem G (2010) Endoscopic approaches to manage in vitro and in vivo embryo development: use of the bovine oviduct. Theriogenology 73: 768-76.

9. Xu J, Chaubal SA, Du F (2009) Optimizing IVF with sexed sperm in cattle. Theriogenology 71: 39-47.

10. Morotti F, Sanches BV, Pontes JH, Basso AC, Siqueira ER, et al. (2014) Pregnancy rate and birth rate of calves from a large-scale IVF program using reversesorted semen in Bos indicus, Bos indicus-taurus, and Bos taurus cattle. Theriogenology 81: 696-701.

11. Kepkova KV, Vodicka P, Toralova T, Lopatarova M, Cech S, et al. (2011) Transcriptomic analysis of in vivo and in vitro produced bovine embryos revealed a developmental change in cullin 1 expression during maternal-to-embryonic transition. Theriogenology 75: 1582-95.

12. Pellegrino CA, Morotti F, Untura RM, Pontes JH, Pellegrino MF, et al. (2016) Use of sexed sorted semen for fixed-time artificial insemination or fixed-time embryo transfer of in vitro-produced embryos in cattle. Theriogenology doi: 10.1016/j.theriogenology.2016.03.010.

13. Stroud B (2011) The year 2010 worldwide statistics of embryo transfer in domestic farm animals. In: IETS 2011 Statistics and data retrieval committee report. Embryo Transfer Newsletter 29: 14-23.

14. Block J, Hansen PJ, Loureiro B, Bonilla L (2011) Improving post-transfer survival of bovine embryos produced in vitro: actions of insulin-like growth factor-1, colony stimulating factor-2 and hyaluronan. Theriogenology 76: 1602-9.

15. Lim KT, Jang G, Ko KH, Lee WW, Park HJ, et al. (2007) Improved in vitro bovine embryo development and increased efficiency in producing viable calves using defined media. Theriogenology 67: 293-302.

16. Neira JA, Tainturier D, Peña MA, Martal J (2010) Effect of the association of IGF-I, IGF-II, bFGF, TGF- $\beta 1$, GM-CSF, and LIF on the development of bovine embryos produced in vitro. Theriogenology 73: 595-604.

17. Zullo G, Albero G, Neglia G, De Canditiis C, Bifulco G, et al. (2015) L-ergothioneine supplementation during culture improves quality of bovine in vitroproduced embryos. Theriogenology 85: 688-97.

18. Räty M, Ketoja E, Pitkänen T, Ahola V, Kananen K, et al. (2011) In vitro maturation supplements affect developmental competence of bovine cumulus-oocyte complexes and embryo quality after vitrification. Cryobiology 63: 245-55.

19. Lucas CG, Remião MH, Komninou ER, Domingues WB, Haas C, et al. (2015) Tretinoin-loaded lipid-core nanocapsules decrease reactive oxygen species levels and improve bovine embryonic development during in vitro oocyte maturation. Reprod Toxicol 58: 131-9.

20. Machado MF, Caixeta ES, Sudiman J, Gilchrist RB, Thompson JG, et al. (2015) Fibroblast growth factor 17 and bone morphogenetic protein 15 enhance cumulus expansion and improve quality of in vitro-produced embryos in cattle. Theriogenology 84: 390-8.

21. Tajik P, Niwa K (1998) Effects of caffeine and/or heparin in a chemically defined medium with or without glucose on in vitro penetration of bovine oocytes and their subsequent development. Theriogenology 49: 771-7.

22. Monaco E, Gasparrini B, Boccia L, De Rosa A, Attanasio L, et al. (2009) Effect of osteopontin (OPN) on in vitro embryo development in cattle. Theriogenology 71: 450-7.

23. Córdova B, Morató R, Frutos C, Bermejo-Álvarez P, Paramio T, et al. (2011) Effect of leptin during in vitro maturation of prepubertal calf oocytes: embryonic development and relative mRNA abundances of genes involved in apoptosis and oocyte competence. Theriogenology 76: 1706-15.

24. Picco SJ, Rosa DE, Anchordoquy JP, Anchordoquy JM, Seoan A, et al. (2012) Effects of copper sulphate concentrations during in vitro maturation of bovine oocytes. Theriogenology 77: 373-81.

25. Sun WJ, Pang YW, Liu Y, Hao HS, Zhao XM, et al. (2015) Exogenous glutathione supplementation in culture medium improves the bovine embryo development after in vitro fertilization. Theriogenology 84: 716-23.

26. Takada L, Junior AM, Mingoti GZ, Balieiro JCC, Cipolla-Neto J, et al. (2012) Effect of melatonin on DNA damage of bovine cumulus cells during in vitro maturation (IVM) and on in vitro embryo development. Res Vet Sci 92: 124-7. 
27. Santana PP, Carvalho CM, da Costa NN, Silva TV, Ramos PC, et al. (2014) Effect of dexamethasone on development of in vitro-produced bovine embryos. Theriogenology 82: 10-6.

28. Walters AH, Eyestone WE, Saacke RG, Pearson RE, Gwazdauskas FC (2005) Bovine embryo development after IVF with spermatozoa having abnormal morphology. Theriogenology 63: 1925-37.

29. Zhao S, Liu ZX, Gao H, Wu Y, Fang Y, et al. (2015) A three-dimensional culture system using alginate hydrogel prolongs hatched cattle embryo development in vitro. Theriogenology 84: 184-92.

30. Holm P, Booth PJ, Schmidt MH, Greve T, Callesen H (1999) High bovine blastocyst development in a static in vitro production system using SOFaa medium supplemented with sodium citrate and myo-inositol with or without serum-proteins. Theriogenology 62: 683-700.

31. Abe H, Yamashita S, Itoh T, Satoh T, Hoshi H (1999) Histochemical and ultrastructural evaluations of cytoplasmic lipid droplets in bovine embryos cultured in serum-free and serum-containing media. Theriogenology 51: 232.

32. Sudano MJ, Paschoal DM, Rascado Tda S, Magalhães LC, Crocomo LF, et al. (2011) Lipid content and apoptosis of in vitro-produced bovine embryos as determinants of susceptibility to vitrification. Theriogenology 75: 1211-20.

33. Leivas FG, Brum DS, Fialho SS, Saliba WP, Alvim MT, et al. (2011) Fetal calf serum enhances in vitro production of Bos taurus indicus embryos. Theriogenology 75: 429-33.

34. Saadeldin IM, Kim B, Lee B, Jang G (2011) Effect of different culture media on the temporal gene expression in the bovine developing embryos. Theriogenology 75: $995-1004$.

35. Isobe O, Ikebata Y, Onitsuka T, Do LTK, Sato Y, et al. (2013) Cryopreservation for bovine embryos in serum-free freezing medium containing silk protein sericin. Cryobiology 67: 184-7.

36. Oliveira e Silva I, Vasconcelos RB, Caetano JV, Gulart LV, Camargo LS, et al. (2011) Induction of reversible meiosis arrest of bovine oocytes using a two-step procedure under defined and nondefined conditions. Theriogenology 75: 1115-24.

37. Lee ES, Fukui Y, Lee BC, Lim JM, Hwang WS (2004) Promoting effect of amino acids added to a chemically defined medium on blastocyst formation and blastomere proliferation of bovine embryos cultured in vitro. Anim Reprod Sci 84: 257-67.

38. Nedambale TL, Dinnyes A, Groen W, Dobrinsky JR, Tian XC, et al. (2004) Comparison of in vitro fertilized bovine embryos cultured in KSOM or SOF and cryopreserved by slow freezing or vitrification. Theriogenology 62: 437-49.

39. Lim KT, Jang G, Ko KH, Lee WW, Park HJ, et al. (2008) Improved cryopreservation of bovine preimplantation embryos cultured in chemically defined medium. Anim Reprod Sci 103: 239-48.

40. Sanches BV, Marinho LS, Filho BD, Pontes JH, Basso AC, et al. (2013) Cryosurvival and pregnancy rates after exposure of IVF-derived Bos indicus embryos to forkolin before vitrification. Theriogenology 80:372-7.

41. Pereira RM, Carvalhais I, Pimenta J, Baptista MC, Vasques MI, et al. (2008) Biopsied and vitrified bovine embryos viability is improved by trans10, cis12 conjugated linoleic acid supplementation during in vitro embryo culture. Anim Reprod Sci 106: 322-32.

42. Pryor JH, Looney CR, Romo S, Kraemer DC, Long CR (2011) Cryopreservation of in vitro produced bovine embryos: effects of lipid segregation and post-thaw laser assisted hatching. Theriogenology 75: 24-33.

43. Cagnone G, Sirard MA (2014) The impact of exposure to serum lipids during in vitro culture on the transcriptome of bovine blastocysts. Theriogenology 81 : $712-22$.

44. Mucci N, Aller J, Kaiser GG, Hozbor F, Cabodevila J, et al. (2006) Effect of estrous cow serum during bovine embryo culture on blastocyst development and cryotolerance after slow freezing or vitrification. Theriogenology 65: 1551-62.

45. Gómez E, Rodríguez A, Muñoz M, Caamaño JN, Hidalgo CO, et al. (2008) Serum free embryo culture medium improves in vitro survival of bovine blastocysts to vitrification. Theriogenology 69: 1013-21.

46. Block J, Bonilla L, Hansen PJ (2009) Effect of addition of hyaluronan to embryo culture medium on survival of bovine embryos in vitro following vitrification and establishment of pregnancy after transfer to recipients. Theriogenology 71: 1063-71.

47. Rasmussen S, Block J, Seidel GE Jr, Brink Z, McSweeney K, et al. (2013) Pregnancy rates of lactating cows after transfer of in vitro produced embryos using X-sorted sperm. Theriogenology 79: 453-61.

48. Ghys E, Dallemagne M, De Troy D, Sauvegarde C, Errachid A, et al. (2015) Female bovine blastocysts are more prone to apoptosis than male ones. Theriogenology 85: 591-600.

49. Liu X, Hu T, Sun W, Hao H, Liu Y, et al. (2015) Comparison of the developmental competence and quality of bovine embryos obtained by in vitro fertilization with sex-sorted and unsorted semen from seven bulls. Livestock Science 181: 263-70.

50. Stewart BM, Block J, Moelli P, Navarette AE, Amstalden M, et al. (2011) Efficacy of embryo transfer in lactating dairy cows during summer using fresh or vitrified embryos produced in vitro with sex-sorted semen. J Dairy Sci 94: 3437-45.

51. Jo HT, Bang JI, Kim SS, Choi BH, Jin JI, et al. (2014) Production of female bovine embryos with sex-sorted sperm using intracytoplasmic sperm injection: efficiency and in vitro developmental competence. Theriogenology 81: 675-82.

52. Li J, Zhu S, He X, Sun R, He Q, et al. (2016) Application of a microfluidic sperm sorter to in vitro production of dairy cattle sex-sorted embryos. Theriogenology 85: 1211-8. 
Submit your next manuscript to Annex Publishers and
benefit from:
$>$ Easy online submission process
$>$ Rapid peer review process
$>$ Online article availability soon after acceptance for Publication
$>$ Open access: articles available free online
> More accessibility of the articles to the readers/researchers within the field
Submit your manuscript at
http://www.annexpublishers.com/paper-submission.php 